\title{
Clinicopathological Study of Canine Monocytic Ehrlichiosis in Kolkata, India
}

\author{
M. Mondal*, S. S. Kesh, A. Maity, S. Palai and D. Mandal \\ Faculty of Veterinary \& Animal Sciences, West Bengal University of Animal \& Fishery \\ Sciences, OUAT, Bhubaneswar, India
}

Keywords

Canine Monocytic

Ehrlichiosis,

Clinicopathology, Kolkata

Article Info

Accepted:

22 July 2019

Available Online:

10 August 2019

\section{A B S T R A C T}

Canine Monocytic Ehrlichiosis is a worldwide tick borne disease. In dogs, it is usually caused by Ehrlichia canis, gram-negative obligatory intracellular bacteria. Clinical signs, haemato-biochemical alterations and microscopical examination of blood smears were conducted in 60 different breeds of dogs of either sex presented to the Veterinary Clinical Complex, West Bengal University of Animal \& Fishery Sciences, Kolkata between March and June 2019. The majority of dogs were presented with anorexia, pronounced lethargy, high rise of temperature and generalized lymphadenopathy. Morulae of Ehrlichia canis were found in blood smears from $12 \mathrm{dogs}$. The highest prevalence was found in Labrador. Many of the dogs had tick infestation and the ticks on dogs were identified as Rhipicephalus sanguineus. The most common haemato-biochemical alterations were marked anaemia, leukocytosis, erythropaenia, thrombocytopaenia, neutrophillia, lymphopaenia and eosinophilia followed by hyperproteinaemia, hypoalbuminaemia, hyperglobulinaemia, hyperbilirubinaemia, elevated activities of AST, ALT and ALKP, decreased increased BUN and creatinine levels. Majority of the dogs reflected a rapid clinical response to doxycycline along with supportive therapy.

\section{Introduction}

Canine Monocytic Ehrlichiosis is a widespread tickborne infection (Rhipicephalus sanguineus, the brown dog tick) of canine caused by Ehrlichia canis, a gram-negative obligatory intracellular bacteria belonging to the family Anaplasmataceae. It is reported to have global distribution, but its prevalence in a particular region requires the presence of the vector, the brown dog tick. The objective of the present study was to record the distribution of canine monocytic ehrlichiosis in different breeds of dogs in Kolkata and correlation with clinicopathology. Clinical signs of this disease vary based on acute, chronic and subclinical phase. Generally, this infection is mainly characterized by high rise of temperature $\left(103-105^{\circ} \mathrm{F}\right)$, anorexia and general weakness accompanied by oedematous swelling in the dependant part, epistaxis and lymphadenopathy (Kottadamane et al., 2017).

\section{Materials and Methods}

The present study was conducted in 60 dogs of different breeds presented to the Veterinary Clinical Complex, West Bengal University of 
Animal \& Fishery Sciences, Kolkata. After thorough clinical examination, the dogs with characteristic signs of ehrlichiosis and thrombocytopaenia were screened by blood smear examination with Giemsa staining and blood samples were subjected to evaluate the detail hemato-biochemical alterations. The blood samples drawn from affected dogs were subjected for hematological studies viz. hemoglobin $(\mathrm{Hb})$, total leukocyte count (TLC), differential leukocyte count (DLC), and total platelet count) using conventional methods, and harvested serum samples were utilized for biochemical estimation viz total protein, albumin, bilirubin, aspartate aminotransferase (AST), alanine aminotransferase (ALT), alkaline phosphatase (ALKP), blood urea nitrogen (BUN) and creatinine by biochemical semi autoanalyser (Transasia Bio-medical Ltd., Solan, HP). Results obtained from blood smear examination and hemato-biochemical alterations were compared and analyzed to draw the conclusive diagnosis.

\section{Statistical analysis}

Data obtained in different parameters were statistically analyzed by one-way analysis of variance at $5 \%$ level of significance using SPSS software.

\section{Results and Discussion}

History, clinical signs and the haematological and biochemical studies and microscopical examination of blood smears were conducted in 60 different breeds of dog of either sex between March and June 2019. The majority of the affected dogs had anorexia, pronounced lethargy, high rise of temperature and generalized lymphadenopathy. Many of the dogs had tick infestation and the ticks on dogs were identified as Rhipicephalus sanguineus. Majority of the dogs reflected a rapid clinical response to doxycycline along with supportive therapy. Out of 60 suspected dogs for ehrlichiosis, based on peripheral thin blood smear examination, 12 (20\%) cases were positive for the morulae of Ehrlichia canis in monocytes. The highest prevalence was found in Labrador.

\section{Parasitological prevalence}

Giemsa stained peripheral thin blood smear examination exhibited $20 \%$ (12/60) positivity for the morulae of $E$. canis. It was found as intracytoplasmic inclusion bodies of varying sizes and shapes in monocytes (Fig. 1). The most of the morulae were detected in monocytes as homogeneous and dense inclusions.

\section{Breed-wise distribution}

The breed-wise distribution of Canine Monocytic Ehrlichiosis was depicted in Table 1. The highest prevalence was recorded in Labrador. Usually, in the study area and within the study period, Labrador and German Shepherd dog (GSD) breeds were presented more to the Veterinary Clinical Complex, Kolkata. Dogs positive by microscopy exhibited clinical signs comprised of fever, congested mucus membrane, tick infestation, anorexia. lymphadenopathy, loss of weight, emesis, respiratory distress, epistaxis, petechiae, depression, bleeding tendency, oedema of legs and lethargy.

\section{Hemato-biochemical alterations}

The present haemato-biochemical study revealed marked anaemia, leukocytosis, erythropaenia, thrombocytopaenia, neutrophillia, lymphopaenia and eosinophilia (Table 2) followed by hyperproteinaemia, hypoalbuminaemia, hyperglobulinaemia, hyperbilirubinaemia, elevated activities of AST, ALT and ALKP, decreased increased BUN and creatinine levels (Table 3). 
Fig.1 Morulae of Ehrlichia canis in monocytes of dogs

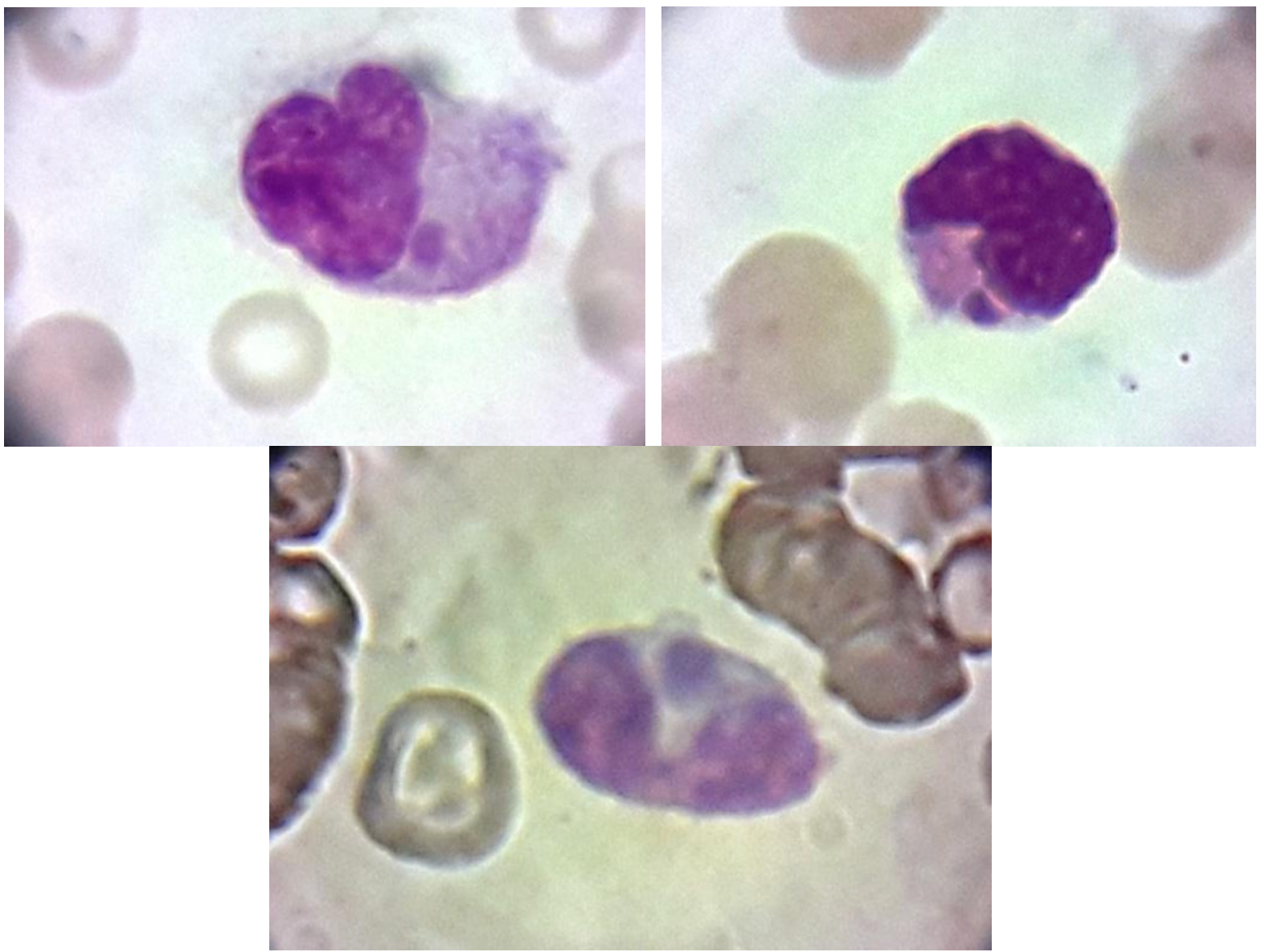

Table.1 Breed-wise distribution of Canine Monocytic Ehrlichiosis

\begin{tabular}{|l|c|c|}
\hline Breed & $\begin{array}{c}\text { Positive in Giemsa } \\
\text { stained blood smear } \\
(\mathbf{n = 1 2})\end{array}$ & Positivity (\%) \\
\hline Labrador & 4 & $33.3 \%$ \\
\hline GSD (German shepherd dog) & 3 & $25 \%$ \\
\hline Pug & 2 & $16.7 \%$ \\
\hline Saint Bernard & 2 & $16.7 \%$ \\
\hline Cocker Spaniel & 1 & $8.3 \%$ \\
\hline
\end{tabular}


Table.2 Haematological alterations of positive dogs for Ehrlichiosis

\begin{tabular}{|l|c|c|}
\hline Parameters & Positive $(\mathbf{n}=\mathbf{1 2})$ & Control $(\mathbf{n}=\mathbf{8})$ \\
\hline Hb $(\mathbf{g} / \mathbf{d L})$ & $8.18 \pm 2.28^{\mathrm{a}}$ & $13.17 \pm 0.67^{\mathrm{b}}$ \\
\hline PCV $(\%)$ & $25.89 \pm 5.73^{\mathrm{ab}}$ & $38.14 \pm 2.72^{\mathrm{c}}$ \\
\hline TEC $\left(\mathbf{1 0}^{\mathbf{6}} / \boldsymbol{\mu L}\right)$ & $4.13 \pm 2.26^{\mathrm{ab}}$ & $6.51 \pm 0.32^{\mathrm{b}}$ \\
\hline TLC $\left(\mathbf{1 0}^{\mathbf{3}} / \boldsymbol{\mu L}\right)$ & $12.35 \pm 6.18^{\mathrm{a}}$ & $7.96 \pm 1.21^{\mathrm{a}}$ \\
\hline Platelet $(\mathbf{1 0} / \boldsymbol{\mu} \mathbf{L})$ & $0.49 \pm 0.28^{\mathrm{a}}$ & $4.03 \pm 0.15^{\mathrm{b}}$ \\
\hline Neutrophils $(\%)$ & $78.43 \pm 13.48^{\mathrm{a}}$ & $73.54 \pm 2.36^{\mathrm{a}}$ \\
\hline Lymphocytes $(\boldsymbol{\%})$ & $20.57 \pm 14.15^{\mathrm{a}}$ & $27.02 \pm 3.53^{\mathrm{a}}$ \\
\hline Eosinophils $(\boldsymbol{\%})$ & $1.48 \pm 2.14^{\mathrm{a}}$ & $2.5 \pm 1.23^{\mathrm{a}}$ \\
\hline
\end{tabular}

Figures with different superscripts in a row differ significantly $(\mathrm{p}<0.05)$

Table.3 Biochemical alterations of positive dogs for Ehrlichiosis

\begin{tabular}{|l|c|c|}
\hline Parameters & Positive $(\mathbf{n}=\mathbf{1 2})$ & Control $(\mathbf{n}=\mathbf{8})$ \\
\hline TP $(\mathbf{g} / \mathbf{d L})$ & $7.46 \pm 1.25^{\mathrm{a}}$ & $6.3 \pm 0.53^{\mathrm{a}}$ \\
\hline Albumin (g/dL) & $2.15 \pm 0.66^{\mathrm{a}}$ & $3.18 \pm 0.33^{\mathrm{b}}$ \\
\hline Globulin (g/dL) & $4.72 \pm 1.49^{\mathrm{b}}$ & $3.32 \pm 0.63^{\mathrm{a}}$ \\
\hline BUN (mg/dL) & $23 \pm 10.56^{\mathrm{a}}$ & $16.3 \pm 4.63^{\mathrm{a}}$ \\
\hline Creatinine (mg/dL) & $1.39 \pm 0.58^{\mathrm{b}}$ & $0.86 \pm 0.31^{\mathrm{a}}$ \\
\hline Total bilirubin (mg/dL) & $0.88 \pm 1.16^{\mathrm{a}}$ & $0.46 \pm 0.12^{\mathrm{a}}$ \\
\hline AST $(\mathbf{U} / \mathbf{L})$ & $27.55 \pm 13.00^{\mathrm{b}}$ & $12.9 \pm 1.33^{\mathrm{a}}$ \\
\hline ALT $(\mathbf{U} / \mathbf{L})$ & $58.17 \pm 27.17^{\mathrm{b}}$ & $26.13 \pm 8.56^{\mathrm{a}}$ \\
\hline ALKP $(\mathbf{U} / \mathbf{L})$ & $133.33 \pm 46.26^{\mathrm{b}}$ & $82.7 \pm 13.15^{\mathrm{a}}$ \\
\hline
\end{tabular}

Figures with different superscripts in a row do not differ significantly $(\mathrm{p}<0.05)$

\section{Blood smear examination and clinical findings}

The present observations of microscopic study were corroborated with the findings of earlier workers (Eljadar, 2010; Dhankar et al., 2011; Shukla et al., 2011). The clinical findings in dogs affected with canine monocytic ehrlichiosis were simulating with the earlier reports (Sacchini et al., 2007; Das and Konar, 2013).

\section{Breed wise distribution}

In the present communication, the disease prevalence was highest in Labrador breed of dogs (Table 1) in comparison to others. Earlier workers also reported that Labrador breed of dogs was most susceptible
(Chandrasekar et al., 2002; Bhadesiya and Modi, 2015; Kottadamane et al., 2017).

\section{Hemato-biochemical findings of ehrlichiosis}

In the present study marked anaemia, leukocytosis, erythropaenia, thrombocytopaenia, neutrophillia, lymphopaenia and eosinophilia (Table 2) followed by hyperproteinaemia, hypoalbuminaemia, hyperglobulinaemia, hyperbilirubinaemia, elevated activities of AST, ALT and ALKP, decreased increased BUN and creatinine levels (Table 3) were the most common observations in diagnosing the canine ehrlichiosis. Bhadesiya and Modi, 2015 also observed significantly deceased mean values of $\mathrm{Hb}, \mathrm{PCV}$, TEC and TLC along with 
marked thrombocytopaenia in canine ehrlichiosis. Sasanelli et al., (2009) also evidenced elevated values of ALT, AST, ALKP, BUN and creatinine accompanied by hyperbilirubinaemia. Asgarali et al., (2012) opined that thrombocytopaenia is the typical characteristic feature of canine ehrlichiosis. Kottadamane et al., (2017) also reported the same biochemical alterations in canine ehrlichiosis. Hypoalbuminaemia and elevated level of ALT activity were also recorded earlier in canine ehrlichiosis (Kottadamane et al., 2017).

The present communication concluded that prevalence and distribution of canine ehrlichiosis in India remain largely unexplored and microscopy was the more useful tool for detecting chronic and clinical infections.

\section{References}

Asgarali, Z., Pargass, I., Adam, J., Mutani, A. and Ezeokoli, C. (2012). Haematological parameters in stray dogs seropositive and seronegative to Ehrlichia canis in north Trinidad. Ticks and Tick Borne Diseases, 3: 207-211.

Bhadesiya, C.M. and Modi, D.V. (2015). Correlation of epidemiology of Rhipicephalous sanguineus and canine ehrlichiosis in nine different localities of middle Gujarat. International Agric. Sc. \& Vet. Med., 3(1): 2320-3730.

Chandrasekar, M., Nambi, A.P., Ramprabhu, R. and Dhanapalan, P. (2002). Epizootiological studies on canine ehrlichiosis. Indian Vet. J., 79(12): 85-
86.

Das, M. and Konar, S. (2013). Clinical and haematological study of canine ehrlichiosis with other hemoprotozoan parasites in Kolkata, West Bengal, India. Asian Pac. J. Trop. Biomed., 3(11): 913-915.

Dhankar, S., Sharma, R.D. and Jindal, N. (2011). Epidemiological observations on canine ehrlichiosis in Haryana and Delhi states. Haryana Vet., 50: 9-14.

Eljadar, M.S.M. (2010). Clinico-Diagnostic Studies on Vector Transmitted Haemoprotozoan Diseases in Dog, M.V.Sc. Thesis, Guru Angad Dev Veterinary and Animal Sciences University, Ludhiana, Punjab.

Kottadamane, M.R., Dhaliwal, P.S., Singla, L.D., Bansal, B.K. and Uppal, S.K. (2017). Clinical and hematobiochemical response in canine monocytic ehrlichiosis seropositive dogs of Punjab. Veterinary World, 10(2): 255-261.

Sacchini, F., Cessford, R.J. and Robinson, B.M. (2007). Outbreak of canine monocytic ehrlichiosis in Saudi Arabia. Vet. Clin. Pathol. 36(4): 33135.

Sasanelli, M., Paradies, P., Lubas, G., Otranto, D. and de Caprariis, D. (2009). Atypical clinical presentation of coinfection with Ehrlichia, Babesia and Hepatozoon species in a dog. Vet. Rec., 164: 22-23.

Shukla, S., Parihar, S., Bariya, S. and Chaturvedi, A. (2011). A clinicpathological report of canine ehrlichiosis in a Doberman pinscher. Vet. World, 4(8): 374-375.

\section{How to cite this article:}

Mondal M., S. S. Kesh, A. Maity, S. Palai and Mandal D. 2019. Clinicopathological Study of Canine Monocytic Ehrlichiosis in Kolkata, India. Int.J.Curr.Microbiol.App.Sci. 8(08): 28732877. doi: https://doi.org/10.20546/ijcmas.2019.808.331 